\title{
A retrospective comparison of cyclophosphamide plus antithymocyte globulin with cyclophosphamide plus busulfan as the conditioning regimen for severe aplastic anemia
}

\author{
L.V.M. Ommati ${ }^{1,2}$, C.A. Rodrigues ${ }^{1}$, A.R. Silva ${ }^{1}$, L.P. Silva ${ }^{1}$, M.L.L.F. Chaufaille ${ }^{1}$ and \\ J.S.R. Oliveira ${ }^{1,2}$
}

${ }^{1}$ Disciplina de Hematologia e Hemoterapia, Departamento de Oncologia Clínica e Experimental, Escola Paulista de Medicina, Universidade Federal de São Paulo, São Paulo, SP, Brasil

2Serviço de Hematologia e Hemoterapia, Hospital Santa Marcelina, São Paulo, SP, Brasil

Correspondence to: J.S.R. Oliveira, Disciplina de Hematologia e Hemoterapia, EPM, UNIFESP, R. Botucatu, 740, 3o andar, 040023-900 São Paulo, SP, Brasil

Fax: +55-11-5571-8806. E-mail: josesro@uol.com.br

\begin{abstract}
Allogeneic hematopoietic stem cell transplantation (AHSCT) is the treatment of choice for young patients with severe aplastic anemia (SAA). The association of antithymocyte globulin (ATG) and cyclophosphamide (CY) is the most frequently used conditioning regimen for this disease. We performed this retrospective study in order to compare the outcomes of HLA-matched sibling donor AHSCT in 41 patients with SAA receiving cyclophosphamide plus ATG (ATG-CY, N = 17) or cyclophosphamide plus busulfan (BU-CY, $N=24$ ). The substitution of BU for ATG was motivated by the high cost of ATG. There were no differences in the clinical features between the two groups, including age, gender, cytomegalovirus status, $A B O$ match, interval between diagnosis and transplant, and number of total nucleated cells infused. No differences were observed in the time to neutrophil and platelet engraftment, or in the risk of veno-occlusive disease and hemorrhage. However, there was a higher risk of mucositis in the BU-CY group (71 vs 24\%, $\mathrm{P}=0.004)$. There were no differences in the incidence of neutrophil and platelet engraftment, acute and chronic graft-versus-host disease, and transplant-related mortality. There was a higher incidence of late rejection in the ATG-CY group (41 vs 4\%, P = 0.009). Although the ATG-CY group had a longer follow-up (101 months) than the BU-CY group (67 months, $P=0.04)$, overall survival was similar between the groups (69 vs $58 \%$, respectively, $P=0.32)$. We conclude that the association $\mathrm{BU}-\mathrm{CY}$ is a feasible option to the conventional ATG-CY regimen in this population.
\end{abstract}

Key words: Aplastic anemia; Allogeneic stem cell transplantation; Conditioning regimen; ATG-CY; BU-CY

Publication supported by FAPESP.

Received June 8, 2008. Accepted January 5, 2009

\section{Introduction}

Allogeneic hematopoietic stem cell transplantation (AHSCT) is the treatment of choice for young adults with severe aplastic anemia (SAA) (1-5), with cure ranging from 75 to $80 \%(5,6)$ and overall survival at 6 years more than $90 \%(7)$.
Many conditioning regimens have been used for SAA with different associations of cyclophosphamide (CY) (6). CY plus antithymocyte globulin (ATG) is the most commonly used regimen due to a low incidence of graft rejection and chronic graft-versus-host disease (GVHD). Improvement in the overall survival for up to $90 \%$ was observed in patients who underwent AHSCT in the first 
months after diagnosis and those who received few blood transfusions prior to transplant $(1,5,6)$.

The first study reporting the use of busulfan (BU) and $C Y$ in a group of highly transfused patients in Brazil showed results comparable to those observed with conventional regimens, resulting in an overall survival rate of $70 \%$ (8). Other Brazilian studies also reported the feasibility of adding a low dose of BU (total dose of $4 \mathrm{mg} / \mathrm{kg}$ ) to $50 \mathrm{mg}$. $\mathrm{kg}^{-1}$. day ${ }^{-1} \mathrm{CY}$ for patients exposed to less than 50 transfusions, with acceptable toxicity $(9,10)$. These issues are especially important in Brazil because less than $15 \%$ of our patients underwent an AHSCT without prior transfusions (6). However, the use of ATG in the conditioning regimen for SAA in Brazil is limited because of its high cost. To the best of our knowledge there are no studies comparing the combination BU-CY and ATG-CY in the conditioning regimen for SAA.

We conducted this retrospective analysis in order to compare the main outcomes of human leukocyte antigen (HLA)-matched related AHSCT for patients with SAA treated with ATG-CY and BU-CY conditioning regimens.

\section{Patients and Methods}

\section{Study group}

From November 1993 to January 2007, 41 patients with SAA underwent an HLA identically matched sibling donor AHSCT at Hospital São Paulo (14 patients) and Hospital Santa Marcelina (27 patients). Diagnosis of SAA was confirmed at admission from the referring center by the Camitta's criteria (11).

HLA class I and II typing was performed by serology and mixed lymphocyte culture before the year 2000, and then by serology and real-time polymerase chain reaction (sequence-specific primers and sequence-specific oligonucleotide probes). All patients received bone marrow stem cells.

All patients or their legal guardians and all donors gave written informed consent for the AHSCT and the study was approved by the Ethics Committee of both Hospitals.

\section{Treatment protocol}

ATG-CY. Treatment consisted of $50 \mathrm{mg} \cdot \mathrm{kg}^{-1} \cdot \mathrm{day}^{-1} \mathrm{CY}$, iv, from day -5 to day -2 , and $30 \mathrm{mg} \cdot \mathrm{kg}^{-1} \cdot \mathrm{day}^{-1}$ horse antithymocyte globulin (UpJohn, USA) from day -4 to day -2 , starting $12 \mathrm{~h}$ after the last dose of CY (12).

$B U-C Y$. Treatment consisted of $12 \mathrm{mg} / \mathrm{kg}$ BU orally in 16 divided doses of $0.75 \mathrm{mg} / \mathrm{kg}$ every $6 \mathrm{~h}$ from day -7 to day -4 , and $60 \mathrm{mg} \cdot \mathrm{kg}^{-1} \cdot \mathrm{day}^{-1} \mathrm{CY}$, iv, on day -3 and day -2 (13). The graft was unmanipulated and GVHD prophylaxis consisted of cyclosporin, administered from day -1 to day +420 and then tapered gradually until day +480 , and $15 \mathrm{mg} / \mathrm{m}^{2}$ methotrexate administered on day +1 , and $10 \mathrm{mg} / \mathrm{m}^{2}$ on days $+3,+6$, and +11 (14).

\section{Supportive care}

Patients were transplanted in private rooms, equipped with HEPA filters. Antimicrobial prophylaxis consisted of trimethoprim-sulfamethoxazole, norfloxacin, fluconazole, and acyclovir. All blood products were irradiated and leukocyte-depleted in both hospitals.

\section{End-point definitions}

Sinusoidal obstruction syndrome was diagnosed according to published criteria (15). Incidence of neutrophil recovery was defined as the first of 3 consecutive days with a neutrophil count of at least $0.5 \times 10 \% / \mathrm{L}$, while platelet recovery was defined as the first of 7 consecutive days of an unsupported platelet count of at least $20 \times 10^{9} / \mathrm{L}$. Graft failure was defined as no sign of neutrophil recovery, as well as only a transient engraftment of donor cells 28 days after transplantation. Acute GVHD at day 100 and chronic GVHD at 5 years was diagnosed and graded according to published criteria (16), with histopathological confirmation when possible. Transplant-related mortality (TRM) at 6 months, 1 and 5 years was defined as deaths related to transplantation and not to relapse.

Chimerism was evaluated by a variable number of tandem repeats, using 4 primers (Apo-B, D1S80, vWF, and DXS52), and also by fluorescent in situ hybridization (FISH), using a double XY probe in sex-mismatched donor-recipient pairs. Chimerism evaluation was performed retrospectively (at least 1 year after the transplant). Full donor chimerism was defined as the presence of more than $99 \%$ of the cells of donor origin, mixed chimerism if more than $1 \%$ and less than $99 \%$ of donor cells and autologous recovery if less than $1 \%$ of donor cells.

\section{Statistical analysis}

Data were analyzed through April 2008. Thus, all surviving patients were followed-up for at least 15 months after transplant. Cumulative incidence function using death as a competing event was used to estimate neutrophil and platelet engraftment, acute and chronic GVHD, rejection and TRM. The Kaplan-Meier method was used to estimate overall survival. For continuous variables, the median was used as the cut-off point. For comparing the groups using cumulative incidence function, univariate analyses were performed using Gray's test (17). For overall survival, logrank tests in univariate analyses were used. $\mathrm{P}$ values $<0.05$ were considered statistically significant. Statistical analyses were performed with SPSS (SPSS Inc., USA) 
and S-Plus (Insightful Corporation, USA) softwares.

\section{Results}

Patient and graft characteristics are shown in Table 1. Median age was 24 years of age, ranging from 14 to 48 years. There were no differences between the groups in most clinical features (age, recipient gender, donor gender, cytomegalovirus (CMV) status, $A B O$ match, number of prior transfusions, time between diagnosis and transplant and number of total nucleated cells infused). In the ATG-CY group, only 3 (18\%) patients were transplanted after 2000, compared with 19 (79\%) in the BU-CY group (P $<0.0001$ ).

The median number of platelet transfusions prior to the transplant was higher in the ATG-CY group (53 units) than in the BU-CY group (13 units, $P=0.06$ ). The number of red blood cell transfusion appeared to be higher in the ATGCY group (20 vs 9 units), but the difference was not statistically significant $(P=0.53)$.

Twenty-four (59\%) patients were treated for SAA before transplant: 10 patients received prednisone alone, 13 received prednisone-based associations with cyclosporine $(\mathrm{N}=5)$, oxymetholone $(\mathrm{N}=5)$, cyclosporine and ATG $(\mathrm{N}=$ $2)$, and cyclosporine and oxymetholone $(N=1)$. One patient received oxymetholone alone.

Median follow-up time of survivors was 75.5 months, ranging from 17 to 152 months. Follow-up was longer for the ATG-CY group (101 months, ranging from 22 to 152) than in the BU-CY group (67 months, range 17-107, $\mathrm{P}=$ $0.04)$. The difference was due to the fact that, after 2000 ,
ATG was no longer available for most patients and we decided to change the standard protocol to BU-CY.

\section{Engraftment and rejection}

Day 30 cumulative incidence of neutrophil recovery was 82 and $75 \%$, and median time to neutrophil recovery was 18 (range, 15-27) and 20 days (range, 13-26) in the ATG-CY and BU-CY groups, respectively $(P=0.22$, Table 2).

Six patients died before day +30 without achieving neutrophil engraftment (4 in the BU-CY group and 2 in the ATG-CY group). Primary graft failure occurred in 2 patients, both in the BU-CY group: 1 patient underwent a second AHSCT from the same donor at day +33 , having received $30 \mathrm{mg} \cdot \mathrm{kg}^{-1}$. day ${ }^{-1} \mathrm{ATG}$, iv, for 3 days as conditioning regimen. The total nucleated cell dose infused was 5.7 $\times 10^{8} / \mathrm{kg}$ in the first transplant and $9.4 \times 10^{8} / \mathrm{kg}$ in the second. He failed to engraft again and died at day +56 due to a pulmonary bacterial infection. The other patient also underwent a second transplant with the same donor at day +35 without receiving any preparative regimen. The total nucleated cell dose was 3.2 and $3.4 \times 10^{8} / \mathrm{kg}$ in the first and second infusions, respectively. He developed acute GVHD and chronic GVHD, and was alive 23 months after the second transplant.

The 100-day cumulative incidence of platelet engraftment was 82 and $67 \%$, and median time to platelet engraftment was 20 (range, 17-33) and 21 days (range, 14-26), in the ATG-CY and BU-CY groups, respectively $(P=0.15)$.

Late rejection occurred in 8 patients. The cumulative incidence of late rejection at 5 years was $41 \%$ in the ATG-

Table 1. Characteristics of patients and grafts.

\begin{tabular}{lrr}
\hline Characteristics & ATG-CY (N =17) & BU-CY (N = 24) \\
\hline Age at transplantation in years, median (range) & $24(14-46)$ & $25(15-48)$ \\
Recipient gender, male, N (\%) & $10(59 \%)$ & $14(58 \%)$ \\
Donor gender, male, N (\%) & $9(53 \%)$ & $9(38 \%)$ \\
Female donor to a male recipient, N (\%) & $4(24 \%)$ & $7(29 \%)$ \\
Recipient CMV positive, N (\%) & $9 / 12(75 \%)$ & $14 / 18(78 \%)$ \\
Donor CMV positive, N (\%) & $7 / 11(64 \%)$ & $13 / 18(72 \%)$ \\
ABO match, N (\%) & $12(71 \%)$ & $15(63 \%)$ \\
Units of red cell transfusions prior to AHSCT, median (range) & $20(0-50)$ & $9(0-97)$ \\
Units of platelet transfusions prior to AHSCT, median (range) & $53(0-180)$ & $13(0-150)$ \\
More than 15 units of red cell transfusions prior to AHSCT & $9 / 16(56)$ & $6 / 23(26)$ \\
More than 20 platelet transfusions prior to AHSCT & $11 / 16(69)$ & $10 / 23(44)$ \\
Interval diagnosis-transplant in months, median (range) & $5(1-18)$ & $2.5(1-74)$ \\
Number of total nucleated cells, $x$ 108/kg, median (range) & $5.1(2.5-9.8)$ & $4.3(2.2-7.9)$ \\
Follow-up time in months, median (range) & $101(22-152)$ & $67(17-107)^{*}$
\end{tabular}

ATG-CY = antithymocyte globulin and cyclophosphamide; $\mathrm{BU}-\mathrm{CY}=$ busulfan and cyclophosphamide; CMV = cytomegalovirus; AHSCT $=$ allogeneic hematopoietic stem cell transplantation. ${ }^{*} \mathrm{P}<0.05$ compared to ATG-CY (Student $t$-test). 
CY group and $4 \%$ in the BU-CY group ( $P=0.009)$. A second transplant was performed in 3 patients: all engrafted after the second transplant. However, one patient had another late graft rejection and died at day +261 from pulmonary aspergillosis. The other two were still alive with a follow-up of 38 and 108 months after the 2nd transplant. The remaining 5 patients were treated with immunosuppression. Four had a good response and are still alive at $11,53,74$, and 81 months after the rejection, and one patient died due to infection and sepsis at day +790 .

Chimerism analysis was performed retrospectively for 16 of $26(62 \%)$ patients who were alive at the time of assessment. Full donor chimerism was observed in 14 (88\%) patients, mixed in one and autologous recovery in one (late graft rejection). The patient with mixed chimerism progressed to graft failure shortly after the chimerism analysis.

\section{Transplant complications}

Mucositis occurred more frequently in the BU-CY group (17 patients, 71\%) compared with the ATG-CY group (4 patients, $24 \%, P=0.004)$, as shown in Table 2 . The incidences of veno-occlusive disease (VOD) and hemorrhage were similar in both groups.

Positive peripheral blood cultures during febrile episode were carried out on 10 occasions. In one of them, in addition to peripheral blood, culture of the central venous catheter was positive. The pathogens that were isolated were Escherichia coli, Enterobacter sp, Staphylococcus coagulase negative, Candida sp (4 cases), Gram-positive coccus, Corynebacterium sp and Pseudomonas sp. Aspergillosis was detected in 4 patients; 1 had bone and 3 pulmonary involvement, 2 of them had fatal disease. Bron- chopneumonia was observed in 9 cases, 5 were fatal. $\mathrm{CMV}$ was diagnosed in 7 cases: positive antigenemia in 5 , and disease in 2 patients, of which one had enteritis and another interstitial pneumonia CMV infection. Varicella zoster infection was observed in 4 cases (one of them presented clinical characteristics of acute abdomen). Possible cerebral toxoplasmosis was diagnosed by MRI images in 1 patient.

\section{Graft-versus-host disease}

Four patients had acute GVHD grade II and 3 patients grade III. The 100-day cumulative incidence of grade II to IV acute GVHD was $6 \%$ in the ATG-CY group and $25 \%$ in the BU-CY group $(P=0.11)$.

Twenty-seven patients were evaluated for chronic GVHD. The 5-year cumulative incidence was $24 \%$ in the ATG-CY group and $22 \%$ in the BU-CY group ( $P=0.96)$. Chronic GVHD was limited in 6 patients and extensive in 3.

\section{Transplant-related mortality}

Fifteen patients $(36.6 \%)$ died from transplant-related causes, 5 in the ATG-CY group and 10 in the BU-CY group. Cumulative incidence of TRM at 5 years was $18 \%$ in the ATG-CY group and $38 \%$ in the BU-CY group ( $P=0.27$ ).

The principal causes of TRM were infections $(N=9)$ : bacterial in 7 cases and fungal in 2. The other causes were: hemorrhage $(N=2), \operatorname{VOD}(N=1)$, late rejection $(N=1)$, chronic GVHD $(\mathrm{N}=1)$, and cardiac toxicity $(\mathrm{N}=1)$.

\section{Overall survival}

Probability of overall survival at 5 years was $69 \%$ for the ATG-CY group and $58 \%$ for the BU-CY group $(P=0.31$, Figure 1).

Table 2. Main treatment outcomes.

\begin{tabular}{lcc}
\hline Characteristics & ATG-CY (N = 17) & BU-CY (N = 24) \\
\hline Time to neutrophil engraftment, median (range) & $18(15-27)$ & $20(13-26)$ \\
Time to platelet engraftment, median (range) & $20(17-33)$ & $21(14-26)$ \\
Development of mucositis, N (\%) & $4(24 \%)$ & $17(71 \%)^{*}$ \\
Development of hemorrhage, N (\%) & $1(6 \%)$ & $6(25 \%)$ \\
Development of VOD, N (\%) & $1(6 \%)$ & $3(13 \%)$ \\
Cl of neutrophil engraftment at 28 days, number of events (\%) & $15(82 \%)$ & $18(75 \%)$ \\
Cl of platelet engraftment at 100 days, number of events (\%) & $15(82 \%)$ & $16(67 \%)$ \\
Cl of acute GVHD at 100 days, number of events (\%) & $1(6 \%)$ & $6(25 \%)$ \\
Cl of chronic GVHD at 5 years, number of events (\%) & $5(22 \%)$ \\
Cl of late rejection at 5 years, number of events (\%) & $4(24 \%)$ & $1(4 \%)^{*}$ \\
Cl of transplant-related mortality at 1 year, number of events (\%) & $7(41 \%)$ & $9(38 \%)$ \\
Probability of overall survival at 5 years, number of events (\%) & $4(24 \%)$ & $10(58 \%)$
\end{tabular}

ATG-CY = antithymocyte globulin and cyclophosphamide; $\mathrm{BU}-\mathrm{CY}=$ busulfan and cyclophosphamide; VOD = veno-occlusive disease; $\mathrm{Cl}=$ cumulative incidence; GVHD = graft-versus-host disease. ${ }^{*} \mathrm{P}<0.05$ compared to ATG-CY (Gray test). 


\section{Discussion}

In this retrospective analysis comparing two conditioning regimens for SAA, outcomes were similar between ATG-CY and BU-CY, except for the incidence of mucositis, which was more frequent with the use of $B U$, and late rejection, surprisingly more frequent with the use of ATG (Table 2).

Since the end of the 1970's, several marrow transplant centers have used $\mathrm{CY}$-based conditioning regimens for SAA (18). At the end of the 1980's, the Seattle group started using the association of CY and ATG, which is currently the most commonly used regimen, with an overall reduction of rejection rates in less transfused patients (12, $13,18,19)$. In Brazil, a pilot study showed that the association of BU and $\mathrm{CY}$ was also feasible in heavily transfused patients (13), with a rejection rate of $9 \%$ and an overall survival of $70 \%$ (8). Since that study, BU-CY has been used by many Brazilian transplant centers due to its lower cost than ATG-CY.

In the present study, ATG was initially available for patients receiving an AHSCT for SAA at both participating centers, but was no longer available after 2001, when it was replaced by busulfan. We then decided to conduct this study in order to compare the main outcomes of the two regimens.

The main clinical features were similar between the two groups. The median number of both platelet and red blood cell transfusions prior to the transplant was higher in the ATG-CY group but this difference was not statistically significant. This may explain the higher rates of graft failure (especially late graft failure) in the ATG group.

Most of the outcomes analyzed were not different between the two groups of patients. Oral mucositis was more frequent and severe in the BU-CY group, as was TRM, probably due to a higher toxicity of $B U$.

VOD, which is not frequently seen in AHSCT for SAA (20), was observed in $9.7 \%$ of the cases, with one case of severe VOD in the BU-CY group. There are only a few reported cases of VOD after AHSCT for SAA and the main reason for this low incidence might be the use of less toxic conditioning regimens, usually based on $\mathrm{CY}$ alone or in combination with ATG (20).

Graft rejection decreased in the ATG-CY era, but it is still frequent, particularly in highly transfused patients $(2,5$, 10). The number of prior transfusions is associated with rejection and survival after AHSCT (10), due to the sensitization by the histocompatibility antigens infused with blood products. In this group, chronic rejection was surprisingly more frequent with the use of ATG than with the use of $B U(P=0.009)$. A possible explanation for this finding is that the ATG group corresponds to transplants performed before the year 2001, when patients were still referred for transplant after having frequently been heavily transfused, and with blood products not leukocyte-depleted in the centers of origin. Leukocyte depletion is routinely used today. Only one case of late rejection was observed in patients submitted to the BU conditioning regimen, which indicates that this regimen is also efficacious in promoting sustained engraftment. This conclusion has been demonstrated in a larger number of patients conditioned with BU-CY (9). Primary and late graft failures were demonstrated in 4/139 (3\%) and 19/135 (14\%), respectively. Therefore, BU-CY should also be considered as an alternative regimen for highly transfused patients (9).

The cumulative incidence of acute GVHD was higher in the BU-CY group (25\%) than in the ATG-CY group (6\%), but the difference was not statistically significant. The cumulative incidence of chronic GVHD in the present study was similar between the ATG-CY and BU-CY regimens (24 and 22\%, respectively), and the majority of patients responded well to immunosuppression (7). Most cases were limited $(\mathrm{N}=6)$, and only one patient died from chronic GVHD. Kröger et al. (19) described an incidence of chronic GVHD of $17 \%$ among patients who underwent an AHSCT for SAA, and only cases of limited disease were observed. Data from the WPSAA (Working Party on Severe Aplastic Anemia) Registry of the EBMT (European Group for Blood and Marrow Transplantation) show an incidence of chronic GVHD of $23 \%$ in SAA, with an observed reduction of

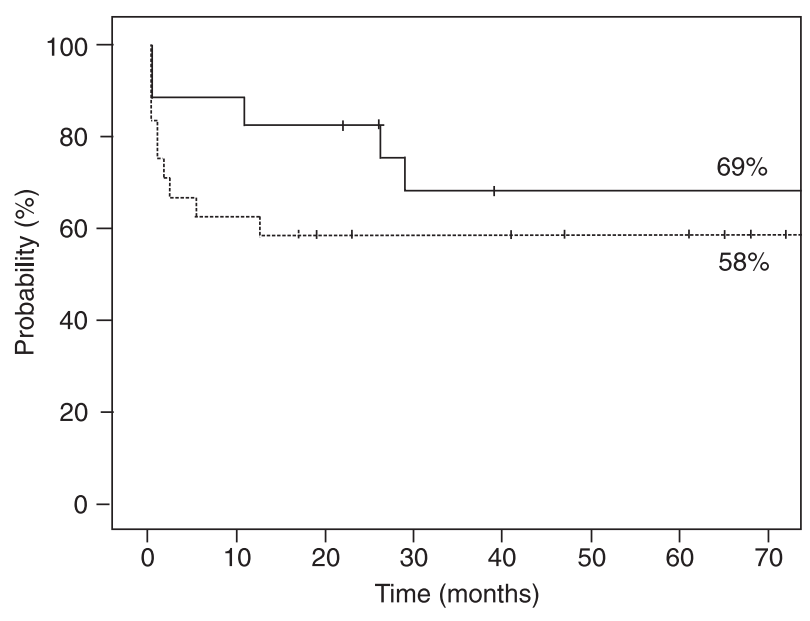

Figure 1. Estimated overall survival according to the conditioning regimen for patients who received the association of antithymocyte globulin and cyclophosphamide (continuous line), or busulfan and cyclophosphamide (dashed line) after allogeneic hematopoietic stem cell transplantation for severe aplastic anemia. There was no statistically significant difference between treatments (log-rank test). 
extensive chronic GVHD of $15 \%$ before 1990 to $5 \%$ in the last decade particularly due to the worldwide use of the CY-ATG association instead of CY plus total body irradiation or thoraco-abdominal irradiation.

Overall survival was slightly higher in the ATG-CY group (69\%) compared to the BU-CY group (58\%), but the difference was not statistically significant. We observed that early deaths were more frequent, probably due to toxicity in the BU-CY group. The main cause of death was infection, which is expected in such a highly immunosuppressed population.

The results in the CY-ATG group are consistent with most previously reported studies, in which overall survival rates varied between 68 and $80 \%(5,21,22)$. More re- cently, a prospective randomized clinical trial $(23,24)$ showed that there was no difference between the ATG-CY association and $\mathrm{CY}$ alone for patients with SAA. All analyzed outcomes (engraftment, GVHD, rejection, and survival) were similar for the two regimens.

The main limitations of the present study were the sample size and the fact that the two regimens were used at different periods of time: ATG-containing regimens were not used after 2001. Larger series of patients with a more homogenous group of patients are needed.

$\mathrm{BU}$ plus $\mathrm{CY}$ is a feasible option in Brazil, due to its relatively low cost compared with ATG-containing regimens.

\section{References}

1. Fonseca $R$, Tefferi A. Practical aspects in the diagnosis and management of aplastic anemia. Am J Med Sci 1997; 313: 159-169.

2. Passweg JR, Socie G, Hinterberger W, Bacigalupo A, Biggs $\mathrm{JC}$, Camitta BM, et al. Bone marrow transplantation for severe aplastic anemia: has outcome improved? Blood 1997; 90: 858-864.

3. Deeg HJ, Leisenring W, Storb R, Nims J, Flowers ME, Witherspoon RP, et al. Long-term outcome after marrow transplantation for severe aplastic anemia. Blood 1998; 91: 3637-3645.

4. Young NS. Acquired aplastic anemia. JAMA 1999; 282: 271-278.

5. Bacigalupo A, Brand R, Oneto R, Bruno B, Socie G, Passweg $\mathrm{J}$, et al. Treatment of acquired severe aplastic anemia: bone marrow transplantation compared with immunosuppressive therapy - the European group for blood and marrow transplantation experience. Semin Hematol 2000; 37: $69-80$

6. Horowitz MM. Current status of allogeneic bone marrow transplantation in acquired aplastic anemia. Semin Hematol 2000; 37: 30-42.

7. Storb R, Blume KG, O'Donnell MR, Chauncey T, Forman SJ, Deeg HJ, et al. Cyclophosphamide and antithymocyte globulin to condition patients with aplastic anemia for allogeneic marrow transplantations: the experience in four centers. Biol Blood Marrow Transplant 2001; 7: 39-44.

8. Pasquini R. Anemia aplástica: transplante de células tronco hematopoéticas. Ser Monogr Esc Bras Hematol 2000; 7: 98-110.

9. Bonfim C, Bitencourt M, Funcke V, Setubal D, Ruiz F, Doro $M$, et al. Bone marrow transplantation (BMT) for heavily transfused patients (pts) with severe aplastic anemia (SAA): 147 pts treated at the same institution with busulfan (BU) + cyclophosphamide (CY). Abstracts from the 2006 BMT Tandem Meetings, February 16-20, 2006, Honolulu, Hawaii. Biol Blood Marrow Transplant 2006; 12 (2 Suppl 1): 1-173.

10. Dulley FL, Vigorito AC, Aranha FJ, Sturaro D, Ruiz MA,
Saboya R, et al. Addition of low-dose busulfan to cyclophosphamide in aplastic anemia patients prior to allogeneic bone marrow transplantation to reduce rejection. Bone Marrow Transplant 2004; 33: 9-13.

11. Camitta BM, Thomas ED, Nathan DG, Gale RP, Kopecky KJ, Rappeport JM, et al. A prospective study of androgens and bone marrow transplantation for treatment of severe aplastic anemia. Blood 1979; 53: 504-514.

12. Storb R, Etzioni R, Anasetti $C$, Appelbaum FR, Buckner CD, Bensinger W, et al. Cyclophosphamide combined with antithymocyte globulin in preparation for allogeneic marrow transplants in patients with aplastic anemia. Blood 1994; 84: 941-949.

13. Pasquini R. Transplante de medula óssea na anemia aplástica. Estudo em 108 pacientes. [Doctoral thesis]. Curitiba: Universidade Federal do Paraná; 1991.

14. Storb R, Deeg HJ, Farewell V, Doney K, Appelbaum F, Beatty $P$, et al. Marrow transplantation for severe aplastic anemia: methotrexate alone compared with a combination of methotrexate and cyclosporine for prevention of acute graft-versus-host disease. Blood 1986; 68: 119-125.

15. Camitta B, O'Reilly RJ, Sensenbrenner L, Rappeport J, Champlin R, Doney K, et al. Antithoracic duct lymphocyte globulin therapy of severe aplastic anemia. Blood 1983; 62: 883-888.

16. Przepiorka D, Weisdorf D, Martin P, Klingemann HG, Beatty P, Hows J, et al. 1994 Consensus Conference on Acute GVHD Grading. Bone Marrow Transplant 1995; 15: 825828.

17. Gray RJ. A class K-sample tests for comparing the cumulative incidence of a competing risk. Ann Statist 1988; 116: $1141-1154$

18. Thomas ED. A history of bone marrow transplantation. In: Blume KG, Forman ST, Appelbaum FR (Editors), Thomas' hematopoietic cell transplantation. 3rd edn. Malden: Blackwell Publishing Ltd.; 2004.

19. Kröger $\mathrm{N}$, Zabelina $\mathrm{T}$, Renges $\mathrm{H}$, Kruger $\mathrm{W}$, Kordes $\mathrm{U}$, Rischewski J, et al. Long-term follow-up of allogeneic stem 
cell transplantation in patients with severe aplastic anemia after conditioning with cyclophosphamide plus antithymocyte globulin. Ann Hematol 2002; 81: 627-631.

20. Lee JH, Lee KH, Choi SJ, Min YJ, Kim JG, Kim S, et al. Veno-occlusive disease of the liver after allogeneic bone marrow transplantation for severe aplastic anemia. Bone Marrow Transplant 2000; 26: 657-662.

21. Paquette RL, Tebyani N, Frane $M$, Ireland $P$, Ho WG, Champlin RE, et al. Long-term outcome of aplastic anemia in adults treated with antithymocyte globulin: comparison with bone marrow transplantation. Blood 1995; 85: 283-290.

22. Doney K, Martin P, Storb R, Whitehead J, Smith A, Hansen
$\mathrm{JA}$, et al. A randomized trial of antihuman thymocyte globulin versus murine monoclonal antihuman T-cell antibodies as immunosuppressive therapy for aplastic anemia. Exp Hematol 1985; 13: 520-524.

23. Socie G, Rosenfeld S, Frickhofen N, Gluckman E, Tichelli A. Late clonal diseases of treated aplastic anemia. Semin Hematol 2000; 37: 91-101.

24. Champlin RE, Perez WS, Passweg JR, Klein JP, Camitta $\mathrm{BM}$, Gluckman E, et al. Bone marrow transplantation for severe aplastic anemia: a randomized controlled study of conditioning regimens. Blood 2007; 109: 4582-4585. 